\title{
A Novel CAZAC Sequence Based Timing Synchronization Scheme for OFDM System
}

\author{
Anuja Das, Biswajit Mohanty, Benudhar Sahu \\ Department of Electronics and Communication Engineering, ITER, SoA University, Bhubaneswar, Odisha, India
}

\begin{tabular}{l} 
Article Info \\
\hline Article history: \\
Received May 26, 2017 \\
Revised Jan 15, 2018 \\
Accepted Feb 2, 2018 \\
\hline
\end{tabular}

\section{Keyword:}

CAZAC

Differential normalization

OFDM

Timing synchronization

\begin{abstract}
Several classical timing synchronization schemes have been proposed for the timing synchronization in OFDM systems based on the correlation between identical parts of OFDM symbol. These schemes show poor performance due to the presence of plateau and significant side lobe. In this paper we present a timing synchronization schemes with timing metric based on a Constant Amplitude Zero Auto Correlation (CAZAC) sequence. The performance of the proposed timing synchronization scheme is better than the classical techniques.
\end{abstract}

Copyright (C) 2018 Institute of Advanced Engineering and Science. All rights reserved.

\section{Corresponding Author:}

Anuja Das,

Department of Electronics and Telecommunication Engineering,

Konark Institute of Science and Technology,

Bhubaneswar, India.

Email: anujadas2006@gmail.com

\section{INTRODUCTION}

In recent times there is an exponential rise in the demand of multimedia wireless services based on broadband standard. A common technology in most of the broadband standards designed to provide broadband services is Orthogonal Frequency Division Multiplexing [1-2]. OFDM is a multicarrier modulation technique, where high data rate serial bits are converted into low data rate parallel paths and the signals in each of the parallel paths modulate orthogonal sub-carriers. This process of transmission of OFDM signal converts frequency selective channel into frequency flat fading channel. In contrast to single carrier communication, OFDM combats the effects of frequency flat fading channel in frequency domain using bank of simple one tap equalizer. Due to the robustness of OFDM to multipath fading, it is adopted in WLAN, DVB-T, LTE-A, MB-OFDM UWB to provide wireless broadband services. However, OFDM is very sensitive of time and frequency synchronization error [3]. In an OFDM system, the timing and frequency of the received OFDM signal should be synchronized with the reference signal at the receiver. Frequency synchronization error in OFDM system results Inter Channel Interference (ICI). Timing synchronization error [2] in OFDM system results in Inter Symbol Interference (ISI), Inter Channel Interference (ICI) and change in the amplitude of the received signal [6].

Several timing synchronization schemes have been reported for OFDM systems which are mostly based on the auto-correlation between identical repeated parts of OFDM symbol. Schimdl et al [3] have proposed a training symbol having two identical sequences. However, the timing metric has a plateau and results in high timing synchronization variance. Subsequently Minn et al [4] have proposed training symbol with repeated sequences and Park et al [5] proposed training symbol with conjugate symmetry sequence. It is observed that the proposed techniques show poor performance due to the presence of significant power in side lobe. Besides PN-sequence [8] CAZAC sequence has a good auto-correlation. So it shows improve frequency 
and timing offset estimation under frequency-selective channel. Fang et al have [7] presented a novel CAZAC sequence based synchronization algorithm for timing and frequency synchronization. Scheme due to Fang at al has a better performance than Park et al[5]. However, the scheme due to Fang et al[7] has poor performance due to presence of side lobe in the timing metric.

Fang et al [7] have designed a symbol with two identical sequence using CAZAC sequence for timing synchronization. However, the timing metric of this technique indicate the presence of side lobe and does not improve the performance significantly.

In this paper we propose a training symbol with repeated symmetric conjugate sequence based CAZAC sequence. We also propose a new timing metric for the timing synchronization utilizing the modified training symbol. Timing metric, main to side lobe ratio and probability of detection is evaluated using simulation. The performance is observed to be better than the previous techniques.

The rest of the paper is organized as follows. Section II presents a brief description of OFDM system followed by proposed method in Section III. Performance of the proposedscheme is presented in Section VI and the paper is concluded in section $\mathrm{V}$.

\section{OFDM SYSTEM DESCRIPTION}

An IFFT operation is carried out on a group of $\mathrm{N}$ symbols to generate time domain OFDM symbol.The $n$th time-domain samples of OFDM signal transmitted through a fading channel is represented as

$$
x[n]=\sum_{k=0}^{N-1} c_{k} e^{j\left(\frac{2 \pi}{N}\right) k n}
$$

where $\mathrm{N}$ is the total number of orthogonal subcarriers, $\mathrm{c}_{\mathrm{k}}$ 's are thek ${ }^{\text {th }}$ complex information symbolwhich modulates $\mathrm{k}^{\text {th }}$ subcarrier. The $n$th received sample from a multipath fading channel having channel impulse response $\mathrm{h}(\mathrm{m})$ is given as

$$
y[n]=\sum_{m=0}^{L-1} h[m] x[n-m], 0<n<N
$$

where L is the memory of the channel. In OFDM system, timing offset is considered as an unknown timing instantof received signal and frequency offset is considered as a phase rotation of the received data in the time domain. Considering these two uncertainties on received signal, the $n$th received signal sample in AWGN channel is given as

$$
r[n]=y\left[n-n_{\epsilon}\right] e^{j\left(\frac{2 \pi \theta_{\in} n}{N}\right)}+w[n]
$$

where, $\mathrm{n}_{\in}$ is the integer-valued unknown arrival time of a symbol$\theta_{\in}$ is the frequency offset and $w(n)$ is the additive white Gaussian Noise (AWGN).

\subsection{OFDM Timing Synchronization Schemes}

It is observed from the received $\mathrm{k}^{\text {th }}$ subcarrier output of OFDM that the output experiences phase rotation, amplitude variation, ICI and ISI due to the presence of timing offset. So, there is a need to estimate timing offset and compensate the estimated timing offset. Several classical timing synchronization schemes such as Schmidl et al, Min et al, Park et al. The scheme due to Schmidl and Cox employ two repeated sequence in one OFDM symbol for timing synchronization and proposed a timing metric based on the correlation between two identical parts of OFDM symbol normalized with the energy of the symbol. However, the timing metric observed to have a plateau with a duration related to cyclic prefix duration. This results in higher mean square error of the timing offset. Subsequently Min et al have proposed a scheme consisting of several repeated parts in an OFDM symbol. The timing metric of this scheme indicate side lobes of higher magnitude and leads to poor timing synchronization performance.

Further enhancements in timing synchronization have been proposed by Park et al [5] and Pang et al [7] which are described below.

1. Park's Scheme: To reduce the side lobe and increase the difference between the peak values of timing metric observed in the scheme due to Min et al, Park et al have proposed a preamble consisting of conjugate and symmetric sequence in an OFDM symbol. The preamble design proposed by Park is given as

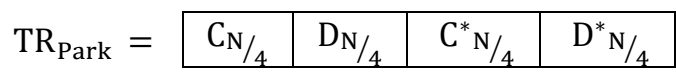


where $\mathrm{C}_{\mathrm{N} / 4}$ represents samples of length $N /{ }_{4}$ generated by IFFT of a PN sequence, and $C^{*}{ }_{\mathrm{N}}{ }_{4}^{\text {represents a }}$ conjugate ofC $\mathrm{N}_{4} \cdot D^{*} \mathrm{~N} / 4$ Symmetricto $D_{\mathrm{N} / 4}$ [3]. The Timing Metric is given by:

$$
\mathrm{M}_{\text {Park }}(\mathrm{d})=\frac{\left|\mathrm{P}_{\text {Park }}(\mathrm{d})\right|^{2}}{\mathrm{R}_{\text {Park }}{ }^{2}(\mathrm{~d})}
$$

where

$$
\begin{aligned}
& \mathrm{P}_{\text {Park }}(\mathrm{d})=\sum_{\mathrm{k}=0}^{\frac{\mathrm{N}}{2}-1} \mathrm{r}(\mathrm{d}-\mathrm{k}) \cdot \mathrm{r}(\mathrm{d}+\mathrm{k}) \\
& \mathrm{R}_{\text {Park }}(\mathrm{d})=\sum_{\mathrm{k}=0}^{\frac{\mathrm{N}}{2}-1}|\mathrm{r}(\mathrm{d}+\mathrm{k})|^{2}
\end{aligned}
$$

Due to impulse-shape timing metric Figure 1, it produces lower Mean Square Error (MSE) in timing offset than Schmidlet al and Min et al [3-4]. In amultipath fading channel, its performances decrease due to the presence of sidelobes. For better performance Fang proposed a method based on CAZAC sequence.

2. Fang Scheme: The correlation based synchronization method is based on auto-correlation property of PN (Pseudo-random Noise)-sequence. Compared to PN sequence, CAZAC sequence has a better autocorrelation and cross-correlation property and hence, improves the timing synchronization performances. The scheme due to Fang assume sequence $\mathrm{s}(\mathrm{k})$ as a CAZAC sequence with length of $\mathrm{N}$ (even number). The Propoerties of CAZAC sequence are

$$
\begin{aligned}
& |s(k)|=\text { Constant, wherek }=0,1,2 \ldots N-1 \\
& \sum_{k=0}^{N-1} s(k) s^{*}(k+\tau)=\left\{\begin{array}{l}
N, \tau=0 \\
0, \tau \neq 0
\end{array}\right.
\end{aligned}
$$

The CAZAC sequence $\mathrm{s}(\mathrm{k})$ described in [8] is written as

$$
s(k)=e^{\left(\mathrm{j}^{\left(\frac{2 \pi \mu \mathrm{k}^{2}}{\mathrm{~N}}\right)}, \mathrm{k}=0,1,2 \ldots \mathrm{N}-1\right.}
$$

where $\mu$ is a positive integer co-prime to $\mathrm{N}$.

Synchronization Preamble Design: The property of CAZAC sequence doesn't change after IFFT operation, so Fang proposed a preamble by repeating CAZAC sequence after IFFT which is shown by below:

\begin{tabular}{|l|l|l|l|l|l|}
\hline $\mathrm{TR}_{\text {Fang }}=$ & $\mathrm{N}_{\mathrm{cp}}$ & $\mathrm{C}_{\mathrm{N} / 2}$ & $\mathrm{D}_{\mathrm{N} / 2}$ & $\mathrm{~N}_{\mathrm{cp}}$ & Data \\
\hline
\end{tabular}

Here $\mathrm{C}_{\mathrm{N} / 2}$ isgiven by $\mathrm{C}_{\mathrm{N} / 2}(\mathrm{i})=\mathrm{v}(\mathrm{i}) \cdot \mathrm{D}_{\mathrm{N} / 2}(\mathrm{i})$, vgiven by ressed by: after IFFTmble by Fang proposed a method based on CAZAC sequence. rect timing but stillwhere $\mathrm{i}=0,1 \ldots \frac{\mathrm{N}}{2}-1, \mathrm{v}(\mathrm{i})=$ $\exp (\mathrm{j} \pi \cdot \mathrm{rn}(\mathrm{i}))$ is a random sequence andrn(i) is the uniformly distributed sequence ranging from-0.2 to 1.Timing Synchronization metric: According to Fang, the timing metric is expressed as

$$
\mathrm{M}_{\text {Fang }}(\mathrm{d})=\frac{\left|\mathrm{P}_{\text {Fang }}(\mathrm{d})\right|^{2}}{\mathrm{R}_{\text {Fang }}{ }^{2}(\mathrm{~d})}
$$

where

$$
\begin{aligned}
& \mathrm{P}_{\text {Fang }}(\mathrm{d})=\sum_{\mathrm{k}=0}^{\frac{\mathrm{N}}{2}-1} \mathrm{v}^{*}(\mathrm{~d}+\mathrm{k}) \cdot \mathrm{r}(\mathrm{d}+\mathrm{k}) \cdot \mathrm{r}^{*}(\mathrm{~d}+\mathrm{k}+\mathrm{N} / 2) \\
& \mathrm{R}_{\text {Fang }}(\mathrm{d})=\frac{1}{2} \sum_{\mathrm{k}=0}^{\mathrm{N}-1}(|\mathrm{r}(\mathrm{d}+\mathrm{k})|)^{2}
\end{aligned}
$$


This scheme gives better performances than Park as it mitigates the sidelobes by multiplication of weighting factor. However, the timing metric indicates the presence of small amount of side lobes. So, we propose a new timing synchronization scheme which reduces the side lobe to almost zero value and improves the probability of detection.

\section{PROPOSED METHOD}

In the proposed method weighted CAZAC sequence is utilized to generate an OFDM symbol with repeated conjugate symmetry sequence. We also propose a new timing metric for timing synchronization based on differential absolute value as a normalized factor.

\subsection{Synchronization Preamble Design}

Our method is the modified version of Park scheme. The training sequence (excluding cyclic prefix), expressed as:

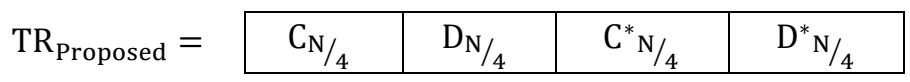

where $C_{N / 4}$ represents first quarter of CAZAC sequence $s(k)$ of length N, i.e., $C_{N / 4}=\exp \left(j \frac{2 \pi \mu k^{2}}{N}\right), k=$ $0,1,2 \ldots\left(\frac{N}{4}-1\right)$ and $D_{N / 4}$ is conjugate and symmetric to $C_{N / 4}$.

\subsection{Timing Synchronization}

In conventional methods, a normalizing factor which is equal to the half energy of the window is used to determine the timing metric. But in our method to get the maximum value we used a different normalization factor which is the difference of absolute value of samples given in (13). The proposed normalization factor is expressed as

$$
\mathrm{R}_{\text {Proposed }}(\mathrm{d})=\sum_{\mathrm{k}=0}^{\frac{\mathrm{N}}{2}-1}(|\mathrm{r}(\mathrm{d}-\mathrm{k})|-|\mathrm{r}(\mathrm{d}+\mathrm{k})|)^{2}
$$

The timing metric based on the difference of magnitude as a normalized factor is given as

$$
\mathrm{M}_{\text {Proposed }}(\mathrm{d})=\frac{\left|\mathrm{P}_{\text {Proposed }}(\mathrm{d})\right|^{2}}{\mathrm{R}_{\text {Proposed }}{ }^{2}(\mathrm{~d})}
$$

where

$$
P_{\text {Proposed }}(d)=\sum_{k=0}^{\frac{N}{2}-1} r(d-k) \cdot r(d+k)
$$

The performance of the schemes due to Park, Fang and the proposed scheme are evaluated using simulation. In Figure 1, the timing metric of each different schemes are plotted where each plot is normalized to their respective maximum value in AWGN channel. Here total subcarrier is taken as 1024; length of CP is 128 samples. From the Figure 1, compare to Park and Fang method, our method has sharper peak and having negligible sidelobes compared to peak value. Therefore, our method has a higher value of probability of detection. 

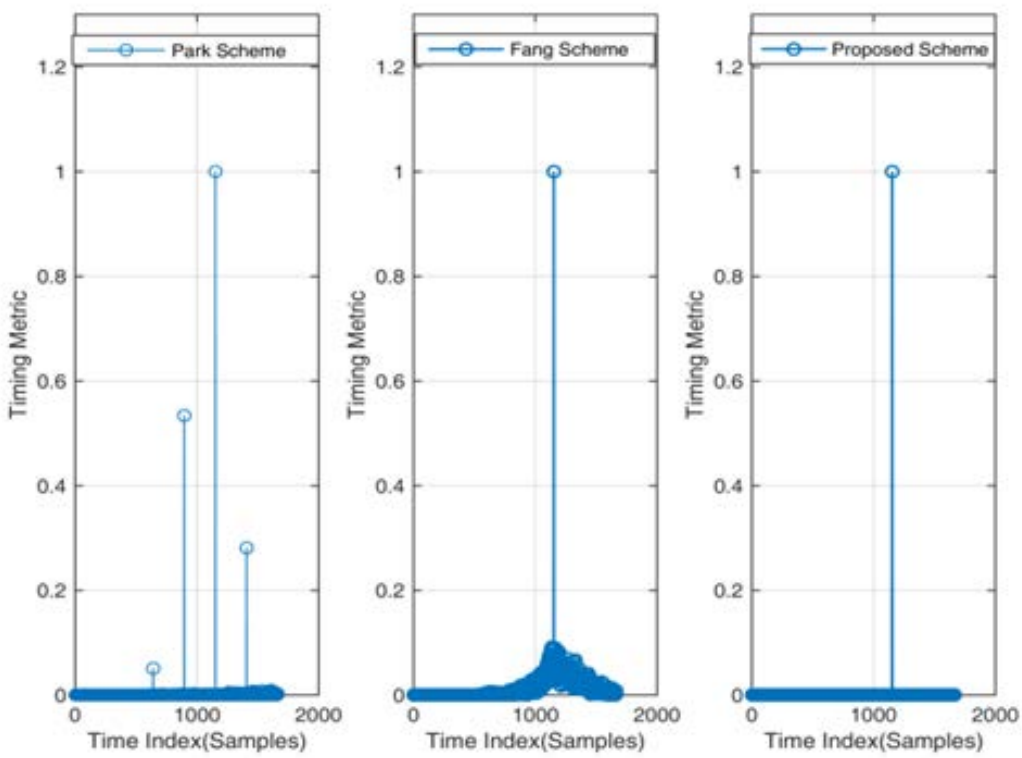

Figure 1. Timing Metric of Different Schemes Normalized to Their Maximum Metric Value

\section{PERFORMANCE EVALUATION}

It is essential to evaluate the performance of the proposed timing synchronization scheme for OFDM system and compare with the classical schemes. We considered an OFDM system with 64 subcarriers $(\mathrm{N})$, cyclic prefix with 16 samples, normalized carrier frequency offset $=0.1$ to evaluate the performance of timing synchronization scheme in exponential decaying multipath fading channel using simulation. The performance metrics which are used to evaluate the performance are, timing metric, Peak to side lobe ratio, Probability of detection.

Figure1 depicts the normalized timing metric for the schemes due to Park et al, Fang et al and the proposed scheme at 10db SNR. It is observed from the timing metric for Park scheme that the side lobe power is of significant magnitude compared to the peak value. However, the timing metric for Fang scheme indicate side lobes with smaller magnitude compared to the peak value. The timing metric for the proposed scheme indicates side lobe magnitude is less than the scheme due to Fang and almost equal to zero.

\subsection{Peak-to-SideLobe Ratio vs. SNR:}

Peak to Side lobe Ratio (PSR) vs SNR in dB is presented in Figure 2. It is observed that the PSR for Park, Fang and the proposed scheme increases with increase in SNR. However, the proposed scheme observed to have a higher PSR of 10 compared to PSR of 3 and 1.75 for Fang and park scheme respectively at $10 \mathrm{~dB}$ SNR.

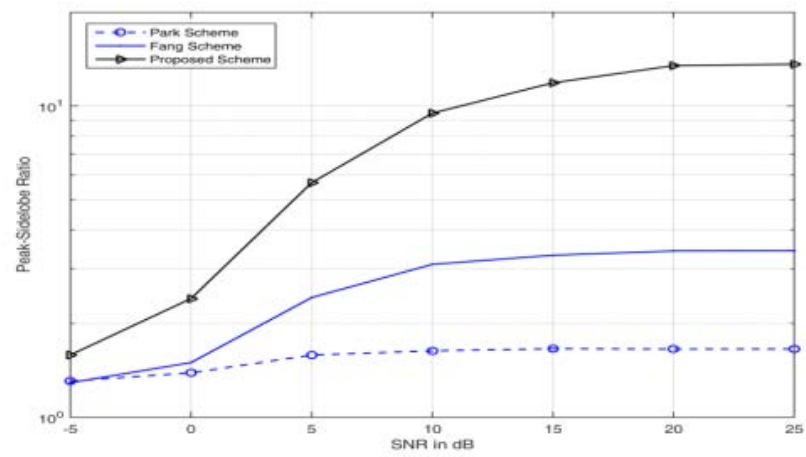

Figure 2. Peak-to-Side Lobe Ratio vs. SNR in dB 


\subsection{Threshold Detection:}

We use the threshold value to detect the timing of the statrt of the symbol. So, there is a need to determine threshold value for the evaluation of probability of detection. We determine the suitable threshold value which will lead to higher probability of detection.To obtain this suitable threshold value we obsereve thevariation of Probability of Detection Failure for different values of threshold.The variation of probability of detection failure vs threshold value at SNR of $10 \mathrm{~dB}$ is shown in Figure 3. It is observed that the suitable threshold value is 0.35 and 0.4 for Fang and Park scheme respectively. However, the threshold value for the proposed scheme is 1.2 .

\subsection{Probability of Detection vs. SNR:}

Probability of Dtection vs SNR is presented in Figure 4. It is observed that the probability of detection remains unchanged till the SNR of $0 \mathrm{~dB}$ for the scheme due to Fang and Park . Further increase in SNR results in sharp rise in probability of detection and it remains unchage at 0.9 for the SNR value beyond $15 \mathrm{~dB}$. However, probability of detection for the proposed scheme indicates a sharp rise with SNR and the probability of detection remain constant at 1 for the SNR beyond $15 \mathrm{~dB}$. It is observed that the probabilty of detection for the proposed scheme is higher than the Park scheme and Fang scheme. Proposed scheme probabilty of detection is about 0.85 compared to 0.25 and 0.1 for Fang and Park scheme respectively at an SNR of $5 \mathrm{~dB}$.

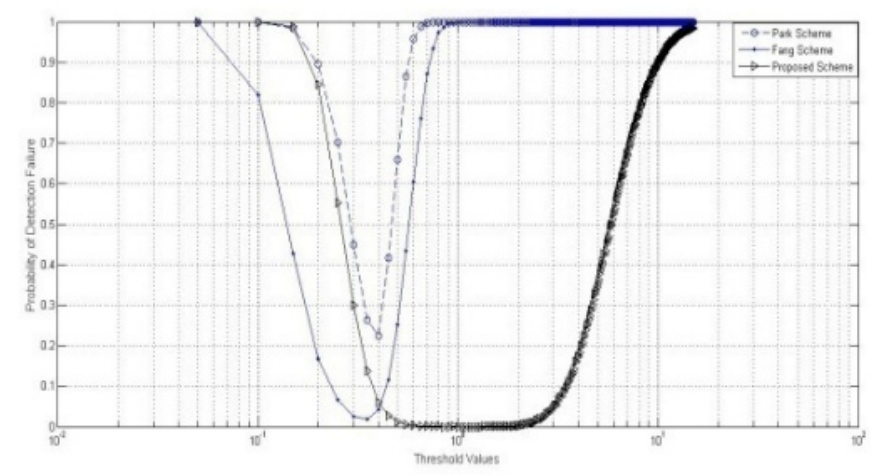

Figure 3.Probability of Detection Failure vs. Threshold Values

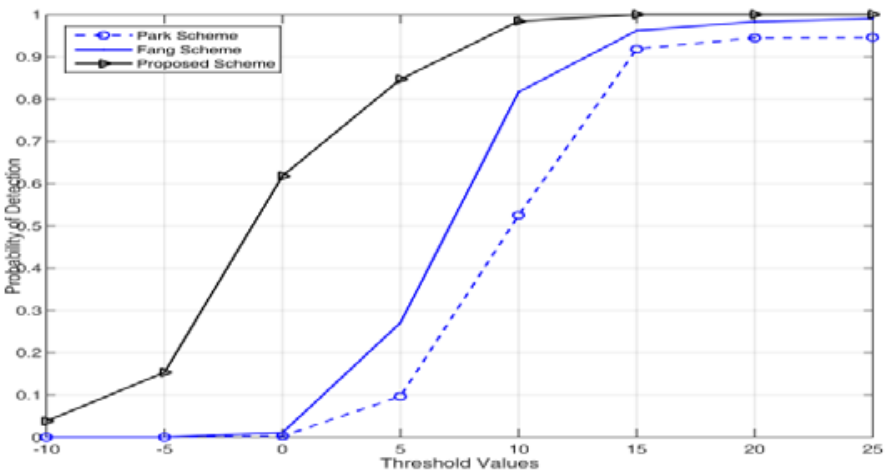

Figure 4. Probability of Detection vs. SNR

\section{CONCLUSION}

In this paper, we proposed a novel timing synchronization method for OFDM system based on CAZAC sequence and differential normalization method, which shows better performances than Park and Fang method. A good correlation property of CAZAC sequence, high impulse-shaped peak at the correcting timing and reduced side lobe makes the proposed method superior than others classical methods. The use of differential normalization allows the system to reduce side lobe and improves the performance. 


\section{REFERENCES}

[1] Van Nee, R.,\&Prasad, R. (2000). OFDM for Wireless Multimedia Communications. Boston, MA: Artech House Publishers.

[2] M. Speth, S. Fechtel, G. Fock, and H. Meyr., ”Optimum Receiver Design for Wireless Broad-Band Systems Using OFDM - Part I,” IEEE Trans. On Comm., 47(11):1668-1677, November 1999.

[3] T. M. Schmidl and D. C. Cox, "Robust frequency and timing synchronization for OFDM," IEEE Trans. Commun., vol. 45, pp. 1613-1621,Dec. 1997.

[4] H. Minn, V. Bhargava, and K. Letaief, “A robust timing and frequency synchronization for OFDM systems,” IEEE Trans. Wireless Commun., vol. 2, no. 4, pp. 822-839, July 2003.

[5] B. Park, H. Cheon, C. Kang, and D. Hong, "A novel timing estimation method for OFDM systems," IEEE Commun. Lett., vol. 7, pp. 239-241,May 2003.

[6] Suyoto Suyoto , I Iskandar, S Sugihartono, Adit Kurniawan, "Improved Timing Estimation Using Iterative Normalization Technique for OFDM Systems ," International Journal of Electrical and Computer Engineering (IJECE), pp.905-911, 2012.

[7] Fang, Yibo, Zhang, Zuotao, Liu Guanghui, “A Novel Synchronization Algorithm Based on CAZAC Sequence for OFDM Systems," International Conference on Wireless Communications, Networking and Mobile Computing, vol., no., pp. 1,4, 21-23, September. 2012.

[8] Han WANG, Leiji ZHU, Yusong SHI , Tao XING , Yingguan WANG, “A Novel Synchronization Algorithm for OFDM Systems with Weighted CAZAC Sequence, ” Journal of Computational Information Systems,pp.2275-2283, 2012. 